\title{
CALCULATION OF AURORAL EMISSION RATES AND HEATING EFFECTS
}

\author{
R. S. STOLARSKI \\ Space Physics Research Laboratory, Department of Electrical Engineering, \\ University of Michigan, Ann Arbor, Michigan
}

(Received in final form 15 May 1968)

\begin{abstract}
The primary electron energy spectrum in an aurora has been determined by fitting the altitude profile of the $3914 \AA$ emission. This spectrum has been degraded through the atmosphere and the secondary electron energy spectra have been calculated at various altitudes. From these spectra the excitation and emission rate profiles of many important lines have been calculated. These include the oxygen $5577 \AA$ and $6300 \AA$ lines and the $\mathrm{N}_{2} 1$ st and 2nd positive and Vegard-Kaplan bands. The heat input to the ambient electrons and the resultant electron temperature have also been calculated.
\end{abstract}

\section{INTRODUCTION}

In a recent paper, Belon, Romick and Rees (1966) determined the incident auroral primary electron energy spectrum by making use of the measured energy dissipation distribution of Grün (1957) and fitting the results to the measured $3914 \AA$ profile in the aurora. In a subsequent paper, Rees, Walker, and Dalgarno (1967) made use of this spectrum to calculate the volume emission rate profiles of the atomic oxygen $5577 \AA$ and $6300 \AA$ lines. The present paper repeats these calculations and extends them to the calculation of the emission rate profiles of other lines in the aurora. This is accomplished by the calculation of the energy spectrum of the secondary electron production rate from which the full knowledge of the volume excitation rates may be obtained and converted to volume emission rates. The heating of the ambient electrons is also obtained along with the resultant electron temperature profile.

\section{DEGRADATION OF PRIMARY SPECTRUM}

The determination of the incident primary energy spectrum from the $3914 \AA$ emission rate profile is made possible by the laboratory measurements of Grün (1957). He measured the energy dissipation rate of electrons in air as a function of depth of penetration for mono-directional electrons of 5-54 keV. This was done by measuring the luminosity, which consisted primarily of $\mathrm{N}_{2}{ }^{+}$1st negative bands and some $\mathrm{N}_{2}$ 1st positive bands, at each point of a two dimensional grid. The luminosity was assumed to be proportional to the ionization rate at that point, which is in turn proportional to the energy dissipation rate. Figure 1 shows the curves of constant luminosity measured by Grün.

Ccrtain assumptions must be made in order to apply these rcsults to the calculation of auroral energy loss. The first assumption concerns the energy range of the experiment. Grün found that the energy dissipation as a function of the fraction of the range (in $\mathrm{g} / \mathrm{cm}^{2}$ ) an electron has traversed, is almost a universal function within the energy limits of 5-54 $\mathrm{keV}$. In the absence of any further data it will be assumed that this 'universal function' is also correct for energies below $5 \mathrm{keV}$.

The second assumption which must be made is that Grün's measurements in air correspond to the actual energy dissipation in the varying constituents of the atmosphere. This 


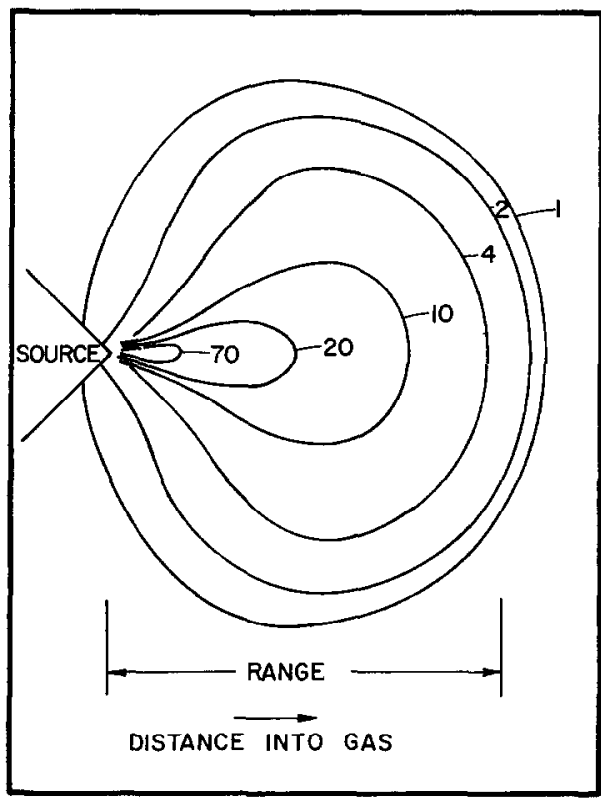

Fig. 1. CuRves of CONSTANT Luminosity. ReProduced fROM GRÜN (1957).

may be easily justified by looking at the energy loss rates of Green and Barth (1967) which show that the energy loss rate per unit mass of gas are virtually the same for $\mathrm{N}_{2}, \mathrm{O}_{2}$, and $\mathrm{O}$.

In order to obtain the energy loss as a function of depth in the gas, Grün simply had to integrate across the luminosity curves at right angles to the beam. In the case of oblique incidence at the top of the atmosphere, this integration at right angles to the beam no longer corresponds to a single altitude, and hence the luminosity curves must be re-integrated along the line of constant altitude in the gas. In this way one obtains the dissipation at each altitude for an incident pitch angle distribution. The amount of backscatter is also affected. It changes from 2 per cent at normal incidence to almost 30 per cent at $75^{\circ}$ pitch angle.

Thus, for each angle, $\theta$, there is a 'universal' energy dissipation function, $\lambda_{\theta}(z / r)$ for all incident electron energies, where $z$ is the depth in the gas in $\mathrm{g} / \mathrm{cm}^{2}$ and $R$ is the electron range in $\mathrm{g} / \mathrm{cm}^{2}$. The dissipation function will be normalized such that

$$
\int_{0}^{1} \lambda_{\theta}(z / R) \mathrm{d}(z / R)=1
$$

Consider a beam of electrons of energy $\varepsilon_{o}$ incident upon a gas at angle $\theta$. When they have traversed a distance of $z \mathrm{~g} / \mathrm{cm}^{2}$ of the gas, their average energy will be

$$
\varepsilon(z)=\varepsilon_{0}\left[1-\int_{0}^{z / R\left(\varepsilon_{0}\right)} \lambda_{\theta}\left(z^{\prime} / R\left(\varepsilon_{0}\right)\right) \mathrm{d}\left(z^{\prime} / R\left(\varepsilon_{0}\right)\right)\right], \quad z \leq R\left(\varepsilon_{0}\right) .
$$

The incident primary spectrum will be assumed to be of the power-exponential form given by Belon, Romick and Rees (1966),

$$
f\left(\varepsilon_{0}\right) \mathrm{d} \varepsilon_{0}=\frac{f_{o}}{\alpha} \varepsilon_{0}^{\gamma} \exp \left(-\varepsilon_{0} / \alpha\right) \mathrm{d} \varepsilon_{0}
$$


The pitch angle distribution will be assumed to be isotropic from $0^{\circ}$ to $80^{\circ}$. To determine the residual primary spectrum at any altitude the incident spectrum is divided into intervals of $0.1 \mathrm{keV}$ and $10^{\circ}$ pitch angle, and each interval is considered separately. Electrons in each interval are considered to have the midpoint pitch angle and the endpoints of each energy interval are mapped into the appropriate energy by means of Equation (2) where the appropriate dissipation function, $\lambda_{\theta}$, must be chosen for each angle $\theta$. The electrons in this incident energy interval are then assumed to distribute themselves uniformly in the energy interval determined by Equation (2). Because the residual primary flux at each altitude is calculated directly from the incident 'top of the atmosphere' flux spectrum, the accumulation of errors of calculating at each altitude from the spectrum of the altitude just above it is avoided.

\section{SECONDARY PRODUCTION RATE SPECTRUM}

The secondary electron production rate spectrum is calculated by using the cross sections vs. energy loss for the ionization continua of $\mathrm{N}_{2}, \mathrm{O}_{2}$ and $\mathrm{O}$. These cross sections and the method of their calculation are given in the papers by Stolarski et al. (1967), Watson et al. (1967), and Stolarski and Green (1967). Basically, the method assumes that if $W \mathrm{eV}$ are lost in a given ionizing collision then this is equal to the sum of the ionization energy, $I$, and the encrgy of the cjected secondary elcetron, $E^{\prime}$. Thus, it is only necessary to know the cross section for ionizing into a state of the continuum between $W$ and $W+\mathrm{d} W$, and then to calculate the excitation rate of this state to determine the rate of production of secondaries between $E^{r}$ and $E^{\prime}+\mathrm{d} E^{\prime}$.

\section{DETERMINATION OF INCIDENT PRIMARY SPECTRUM}

From the secondary production rate altitude profile, the $3914 \AA$ emission rate profile is obtained simply by taking the production of secondaries from ionization of $\mathrm{N}_{2}$ and multiplying by the constant fraction 0.07 (McConkey, Woolsey and Burns, 1967; Rapp and Englander-Golden, 1965). The contribution to $3914 \AA$ radiation from secondary excitation was also included and was about 10 per cent of the primary excitation. The parameters for the incident spectrum in Equation (3) are obtained, by starting with an initial guess and calculating the $3914 \AA$ profile as described above. The parameters are then varied until a reasonable fit to data is obtained.

The aurora considcred in the rest of this paper is the same one used by Belon, Romick and Rees (1966) for the bulk of their calculations. It occurred on February 26, 1960 above Ft. Yukon, Alaska. The parameters thus obtained for the power exponential spectrum are $f_{o}=1.73 \times 10^{11}, \gamma=0.5$, and $\alpha=0.8 \mathrm{keV}$. These are not the same parameters obtained by Belon, Romick and Rees (1966). Their spectrum is much broader than the spectrum obtained in this paper. The narrower spectrum seems to be supported by recent auroral measurements of Evans (1967a, b) and Albert (1967). They find sharp spectra peaking between 4 and $10 \mathrm{keV}$. The spectrum of this paper peaks at the relatively low value of $0.4 \mathrm{keV}$ which could be a result of the time of the particular aurora which occurred earlier in the night $(2100 \mathrm{hr})$ than those measured by Evans and Albert.

Figure 2 shows the incident primary flux spectrum and the residual primary flux spectrum at a few points as it passes through the atmosphere. Figure 3 shows how the incident energy of near normal incidence electrons varies as they penetrate into the atmosphere. The fact that the energy differences between any two of these varies by less than a factor of 2 indicates that the energy intervals of the incident spectrum do not spread appreciably as the electrons degrade through the atmosphere. 


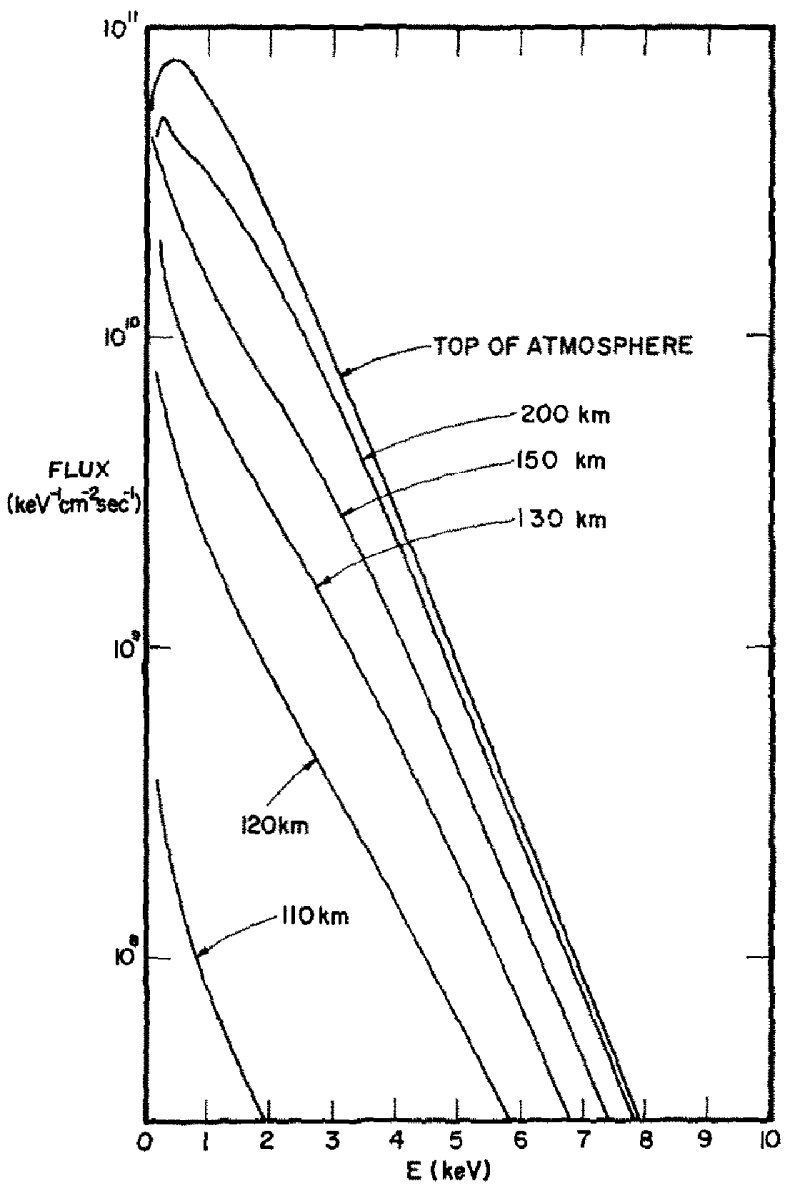

Fig. 2. Calculated Residual PRIMARY FLUX SPECTRUM UNDER THE CONDITIONS OF THE AURORA of February 26. 1960, Above Ft. Yukon, Alaska.

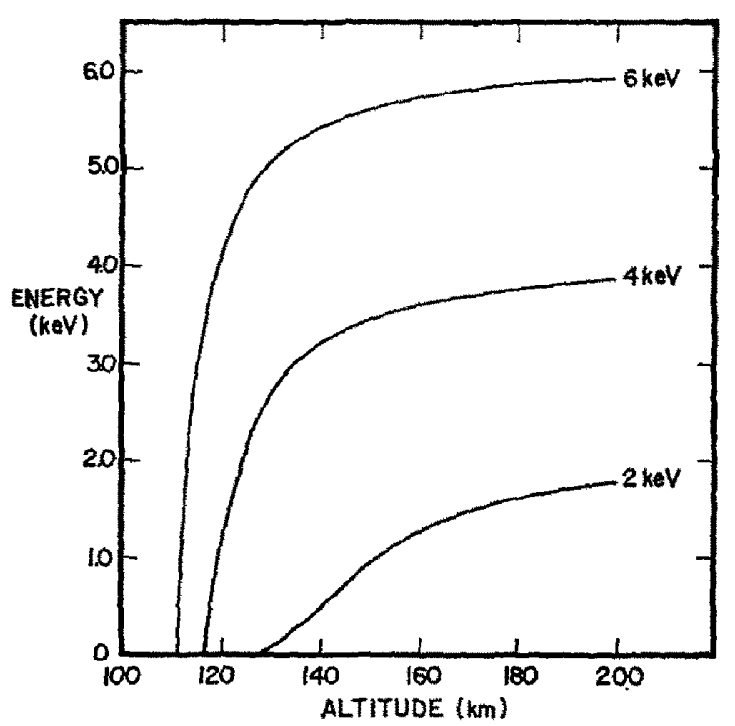

Fig. 3. ENergy of a single electron as a function of altrudue. 


\section{DEGRADATION OF SECONDARIES AND VOLUME EXCITATION RATE PROFILES}

The key quantity which is now available is the energy spectrum of the secondary production rate. Figure 4 shows these for a few sample altitudes. Making the approximation that the secondaries lose their energy locally, which is certainly a reasonable assumption below $200 \mathrm{~km}$, the rate of excitation of any state of the atoms and molecules of the neutral

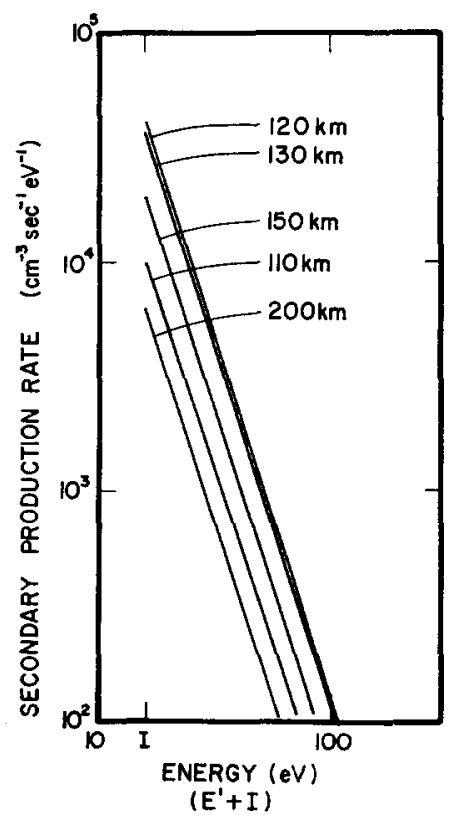

Fig. 4. ENERGy SPECTRA OF THE SECONDARY ELECTRON PRODUCTION RATE.

gas may be readily calculated. The rate of excitation is calculated using the method described by Stolarski and Green (1967). The excitation rate due to a secondary electron of energy $E_{0}$ slowing to zero is

$$
n_{j}=\int_{W_{j}}^{E_{0}} \frac{\sigma_{j}(E) \mathrm{d} E}{\sum_{i} \overline{w_{i} \sigma_{i}(E)}}
$$

where $n_{j}$ denotes the excitation rate of the $j$ th state, $\sigma_{j}(E)$ is the cross section for excitation from the ground state to the $j$ th state and $\overline{w_{i} \sigma_{i}(E)}$ is the average of the energy loss times the cross section.

The excitation rate of a state due to the primary beam may also be calculated using

$$
R_{j}=\int \Phi(E) n \sigma_{j}(E) \mathrm{d} E
$$

where $\Phi(E)$ is the residual primary flux, and $n$ is the number density of the specics involved.

Figure 5 shows the volume excitation rates due to secondaries while Fig. 6 shows the volume excitation rate due to primaries vs. altitude. The reason for the difference in the method of calculation of the excitation rates for primaries and secondaries is that the steadystate flux of primaries is known while only the volume production rate of secondaries is known which means that the loss to the other species must be considered in calculating the excitation rate of a given state. The loss to other species is already taken into account in the derivation of a steady-state flux. 


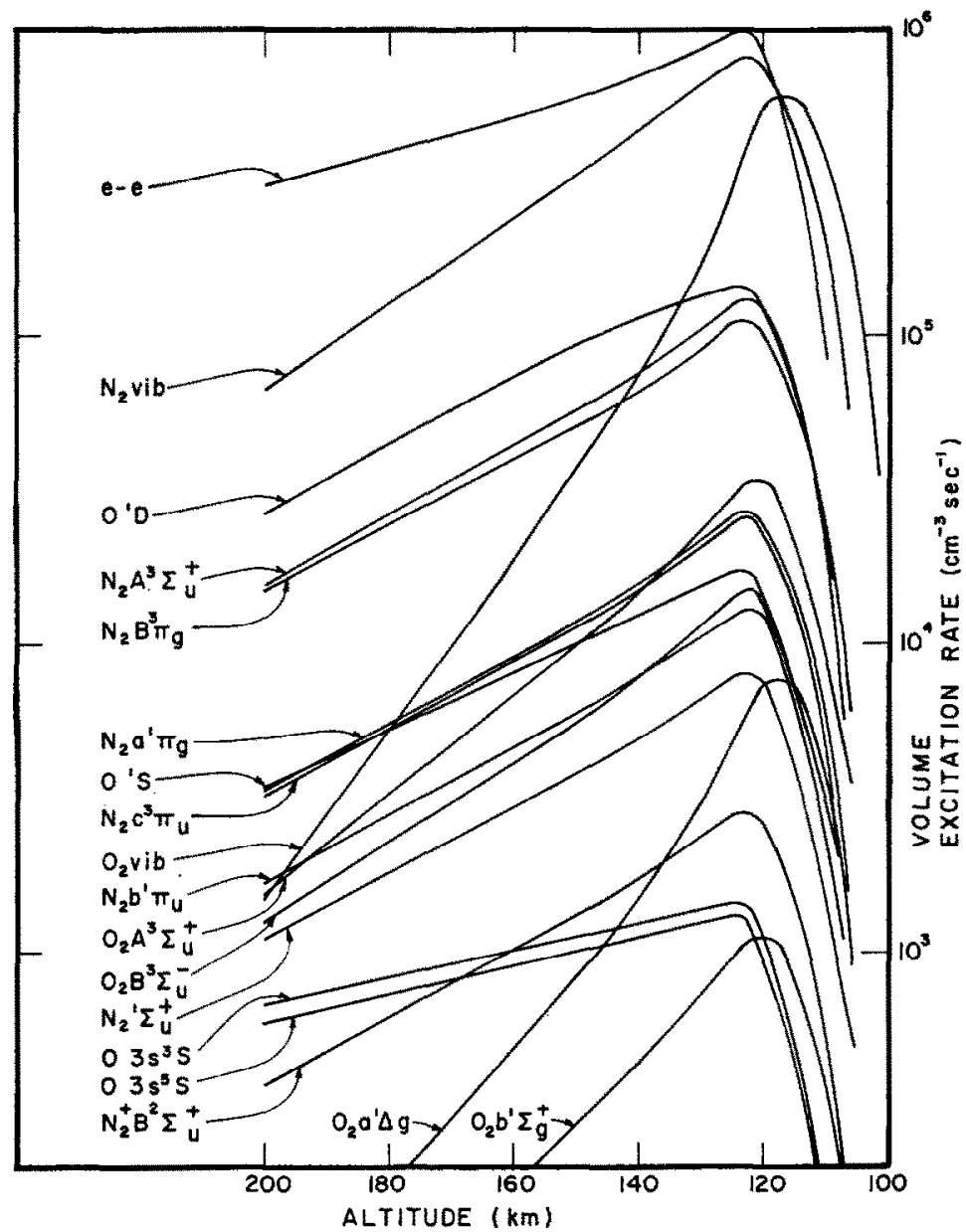

Fig. 5. VOLUME EXCTTATION RATES DUE TO SECONDARY ElECTRONS.

\section{EMISSION RATES}

The volume excitation rates calculated above may now be converted to volume emission rates by consideration of cascading and quenching. Figure 7 shows the calculated $3914 \AA$ radiation coming from the $\mathrm{N}_{2}{ }^{+}\left(B^{2} \sum_{g}+\right.$ state and the comparison with experimental data for this aurora. This is, of course, the so-called 'standard' line with which the incident spectrum was adjusted until an adequate fit was obtained. The differences in the calculated and experimental emission rates reflect only the inexactness of the adjustment of the incident spectrum and the possible errors in the degradation calculation.

Figure 8 shows the volume emission rate of $\lambda 5577$ from the $O\left({ }^{1} S\right)$ state due to direct excitation along with the volume emission rate due to dissociative recombination calculated by Rees, Walker and Dalgarno (1967). The total is then shown in comparison with the experiment for this aurora.

Figure 9 shows the volume excitation rates for the $O\left({ }^{1} D\right)$ state both from direct excitation and from cascading from the $\mathrm{O}\left({ }^{1} S\right)$ state. The total is then corrected for quenching using the coefficients of Hunten and McElroy (1966) to obtain the volume emission rate shown. 


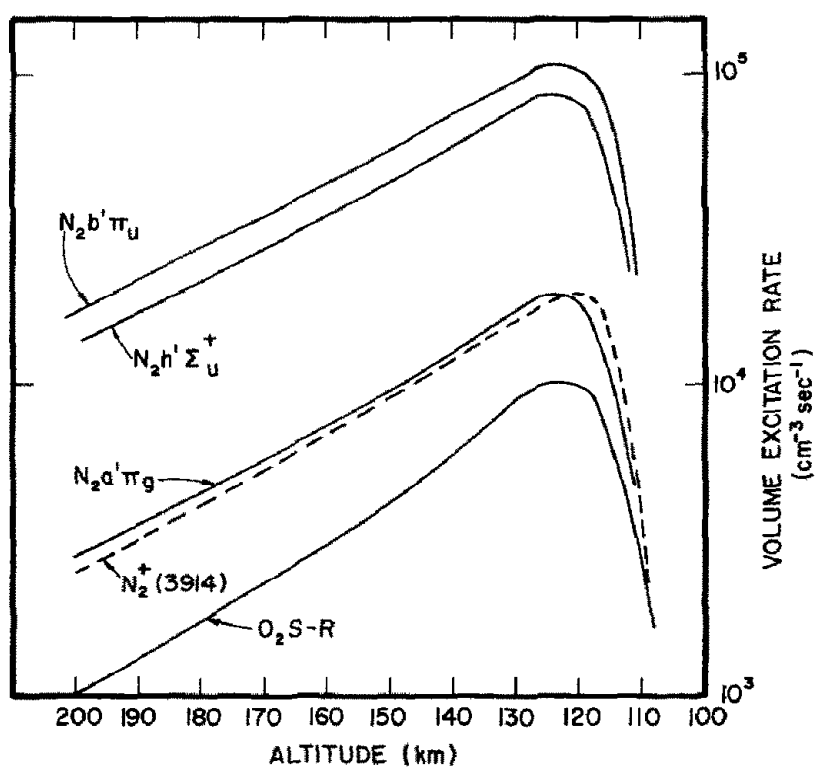

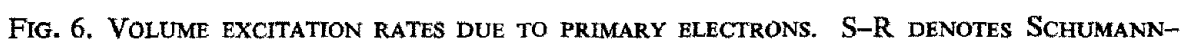
RUNGE CONTINUUM.

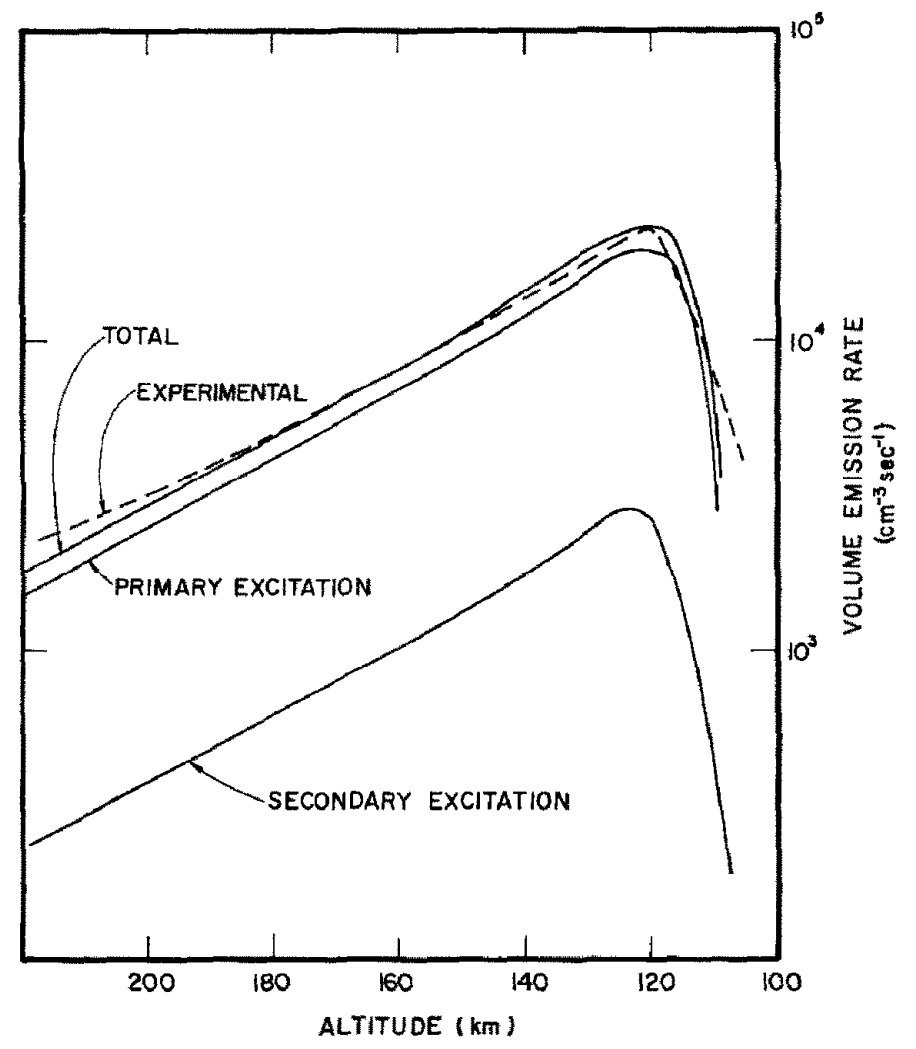

FIG. 7. EXCITATION AND EMISSION RATES OF $\lambda 3914$. 


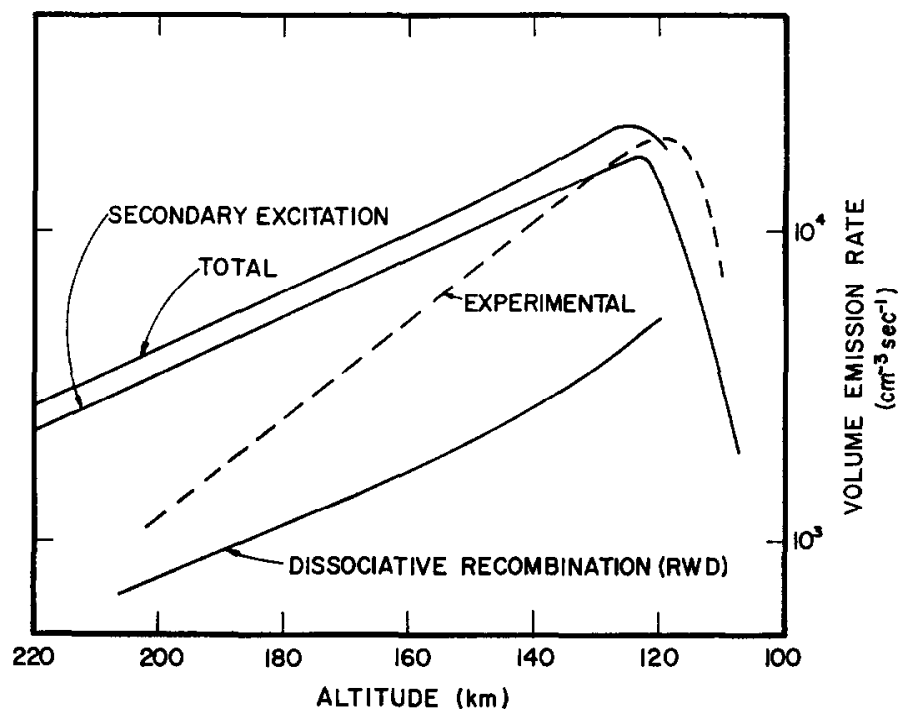

Fig. 8. Excttation and emission rates of 75577 . RWD denotes ReEs, WaLKer and Dalgarno (1967).

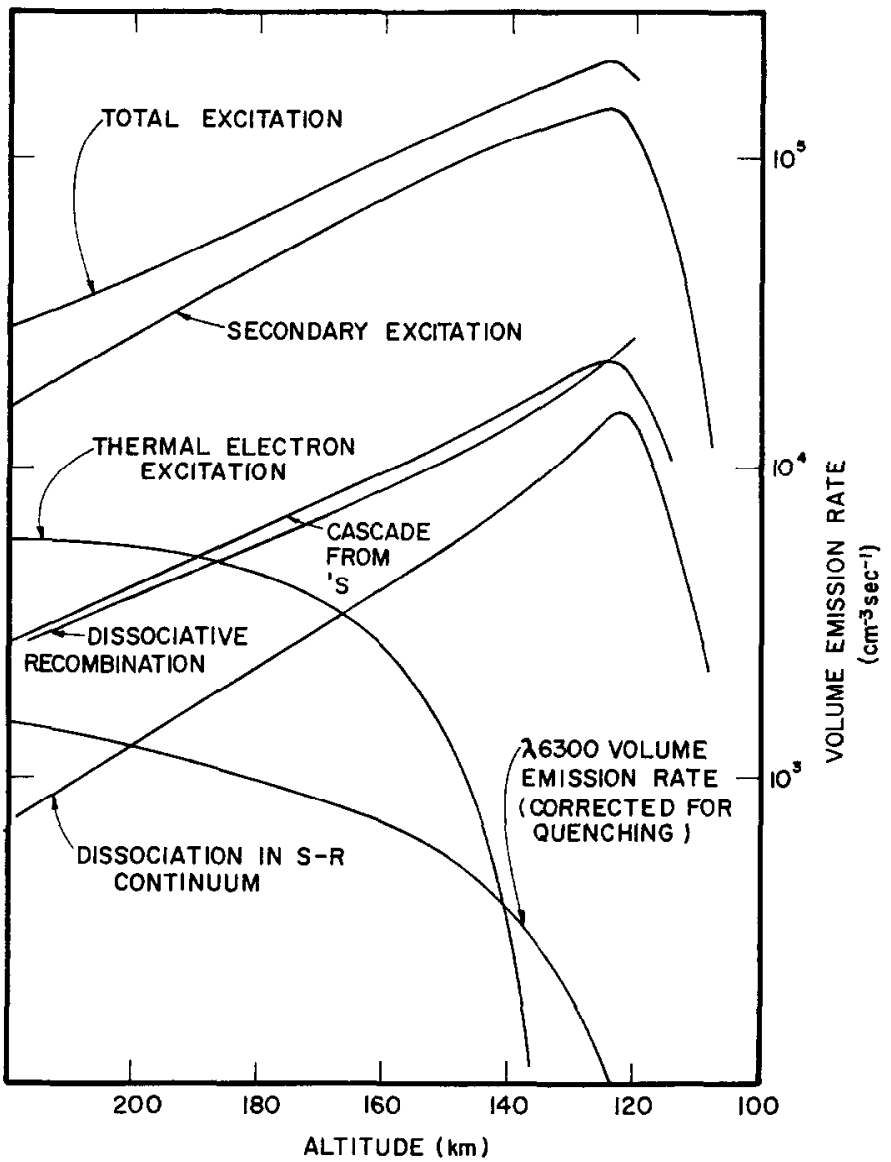

Fig. 9. Excitation and emission rates of $\lambda 6300$. S-R Denotes SChumann-Runge conTINUUM. 


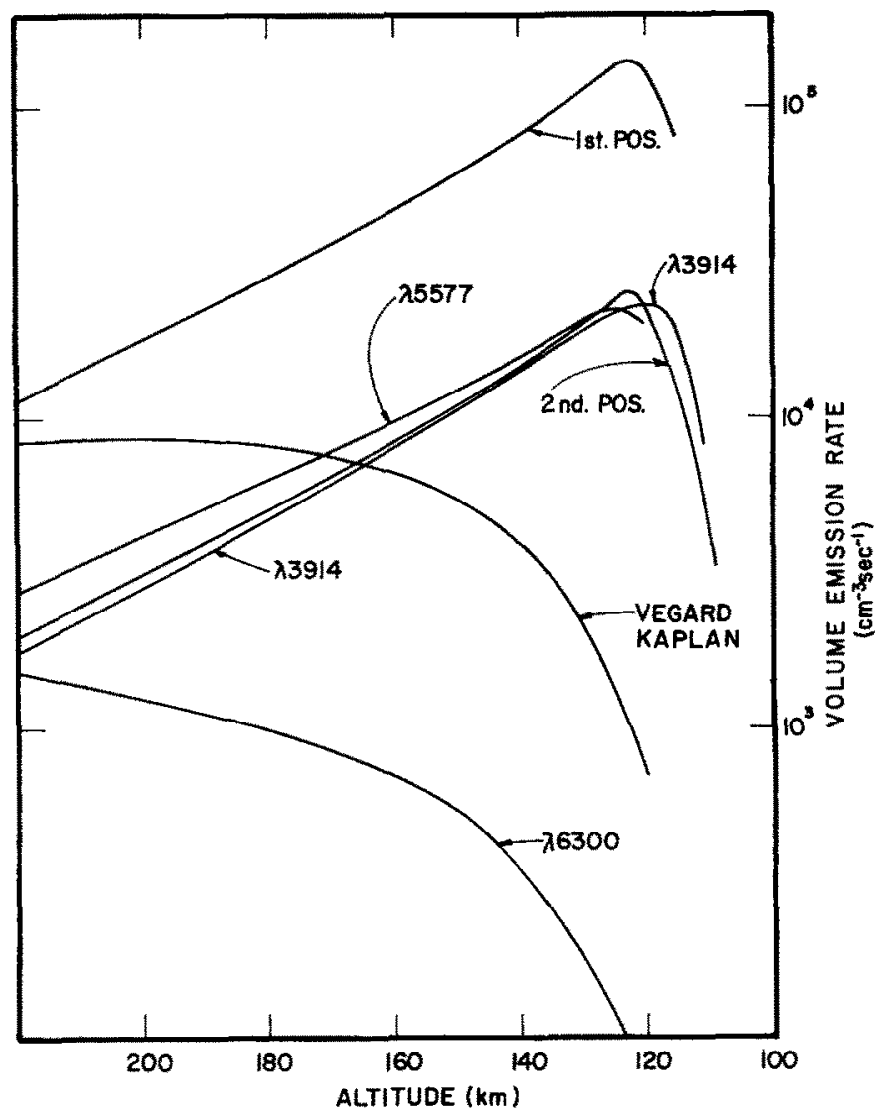

Fig, 10. Summary of Volume emission Rates.

Figure 10 shows a summary of a number of the resultant volume emission rates corrected for cascading and quenching where necessary.

\section{AMBIENT ELECTRON HEATING}

Part of the degradation calculation for the secondaries involved the energy loss to the ambient electron gas obtained by using the table of loss rates tabulated by Dalgarno, McElroy and Moffet (1963) from the equations of Butler and Buckingham (1962). Dalgarno, McElroy and Moffet give an approximation to the loss rate for energies above $3 \mathrm{eV}, \mathrm{A}$ modification of this approximation which was obtained by fitting the exact loss rate curve (for $T_{e}=1500^{\circ} \mathrm{K}$ ) down to a few tenths of an $\mathrm{eV}$ is given by the expression

$$
\frac{1}{n} \frac{\mathrm{d} E}{\mathrm{~d} x} \simeq \frac{1.95 \times 10^{-12}}{E}\left(1-\frac{0.15}{E}\right)^{1 / 2} \text {. }
$$

By using the heat input calculated from this loss rate and the calculated electron density shown in Fig. 11 the conduction equation for the electrons was solved numerically by a slight modification of the method of Dalgarno, McElroy and Walker (1967) to obtain the electron temperature profile in the aurora. The modification includes the oxygen fine structure cooling of the electrons as given by Dalgarno and Degges (1968). Figure 12 shows 


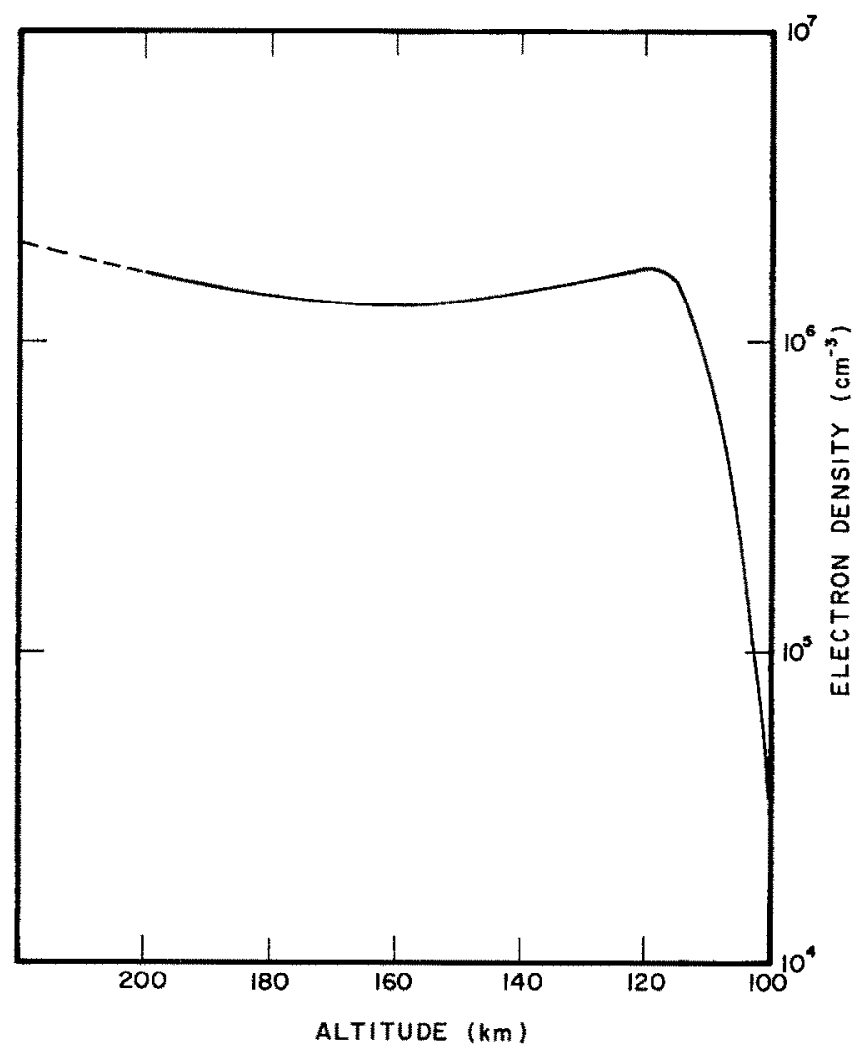

Fig. 11. CalCUlated electron DeNSTTY PROFIL UNDER THE CONDITIONS OF THE AURORA OF February 26, 1960, above Fr. Yukon, Alaska.

the heat input to the ambient electrons while Fig. 13 shows the calculated electron temperature profile.

\section{DISCUSSION}

The differences in the present results and those arising from the papers of Rees (1964a, b) should be reemphasized. The primary spectrum obtained in this paper is much

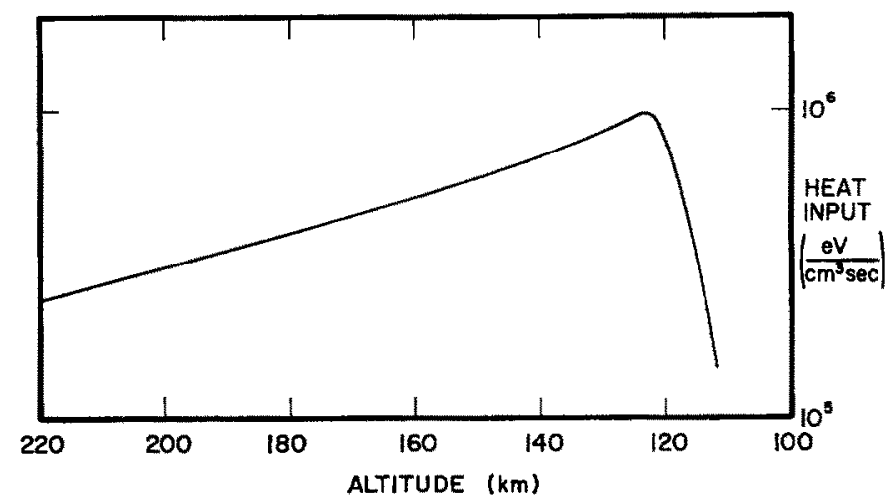

Fig. 12. HeAt INPUT PROFILE TO THE AMBIENT ELECTRON GAS DURING THE AURORA OF FEBRUARY 26. 1960, ABOVE Fr. Yukon, Alaska. 


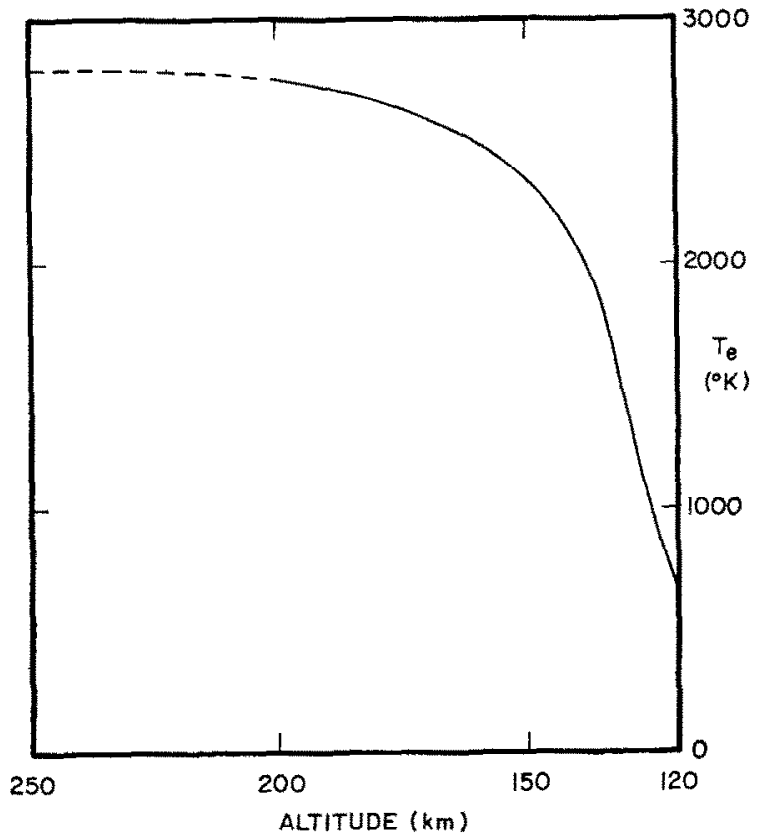

Fig. 13. Calculated electron temperature profile for the heat input presented in FIG. 12 .

narrower and peaks at a lower energy. The emission rate profiles are seen to be very similar because they are actually based on the $3914 \AA$ emission rate profile which in both cases is a fit to the same experimental data.

The reason for the different spectra obtained in the present paper and in the Belon, Romick and Rees (1966) paper is probably the cumulative effect of numerous small differences. First and most obvious of these differences is in the model atmosphere used. Belon, Romick, and Rees' atmosphere has higher densities at all altitudes and consequently needs a harder primary spectrum to give the same intensities at the lower altitudes. Also, there are always the possible variations in the integration of Grün's luminosity curves at different angles. Then, there is the difference in method employed. In the present paper the secondary energy spectrum was calculated in order to determine emission rate profiles of many lines not previously calculated. In doing so, the 3914 emission rate was calculated by going through the step of calculating the residual primary spectrum which was not necessary in the Rees (1963) paper. It should be pointed out, though, that Rees (1964) did calculate the residual primary spectrum in connection with his calculations relating to bremsstrahlung. His residual primary flux differs from the present result. The difference is that in the integral of the dissipation function

$$
\int_{0}^{z / R} \lambda\left(z^{\prime} / R\right) \mathrm{d}\left(z^{\prime} / R\right)
$$

which represents the fraction of the energy lost by the beam in traversing $z \mathrm{~g} / \mathrm{cm}^{2}$, Rees (1964) considered that this fraction of the electrons were absorbed while the rest continued unchanged in energy. In the present paper each electron was considered to have lost this fraction of its energy and thus slowed down as it penetrated the atmosphere. This is probably a more realistic assumption. 
The real problem is the relative insensitivity of the 3914 profile to the incident spectrum. If this is to be overcome, the approximations and assumptions presently inherent in the calculation must be improved or avoided.

The important points of this paper are the calculation of the residual primary spectrum as a function of altitude and from that the calculation of the energy spectrum of the secondary production rate. From these the excitation of any state of the atmospheric atoms and molecules could be determined. The excitation and emission rates for most of the important auroral lines have been calculated and presented.

Acknowledgements - The author would like to acknowledge the many helpful comments and suggestions of Drs. E. G. Fontheim and A. F. Nagy. He would also like to thank Drs. P. B. Hays, J. C. G. Walker and Newbern Smith for helpful discussions concerning the paper. Special thanks go to Dr. J. C. G. Walker for kindly providing his computer program for the electron temperature. Support for this project was provided by National Aeronautics and Space Administration grant NsG-525 and National Science Foundation grant GA1025.

\section{REFERENCES}

AlberT, Richard D. (1967). Energy and flux variations of nearly monocnergetic auroral electrons. $J$. geophys. Res. 72, 5811.

BeLON, A. E., G. J. Romick and M. H. ReEs (1966). The energy spectrum of primary auroral electrons determined from auroral luminosity profiles. Planet. Space Sci. 14, 597.

Butler, S. T. and M. J. Buckingham (1962). Energy loss of a fast ion in a plasma. Phys. Rev. 126, 1.

Dalgarno, A. and T. C. DegGes (1968). Electron cooling in the upper atmosphere. Planet. Space Sci. 16, 125.

Dalgarno, A. M. B. McElroy and R. J. Moffett (1963). Electron temperatures in the ionosphere. Planet. Space Sci. 11, 463.

Dalgarno, A., M. B. MCElroy and J. C. G. Walker (1967). The diurnal variation of ionospheric temperatures. Planet. Space Sci. 15, 331.

Evans, David S. (1967). A 10-cps periodicity in the precipitation of auroral-zone electrons. J. geophys. Res. 72, 428.

Evans, DAvid S. (1967). Rocket observations of low energy auroral electrons. In Aurora and Airglow (Ed. Billy M. McCormac), Reinhold, New York.

GREEN, A. E. S. and C. A. BARTH (1967). Calculations of the photoelectron excitation of the dayglow. J. geophys. Res. 72, 3975 .

GRÜN, A. E. (1957). Lumineszenz-photometrische Messungen der Energieabsorption in Strahlungsfeld von Elektronquellen. Eindimensionaler Fall in Luft. Z. Naturf. 12a, 89.

MCConKEY, J. W., J. M. Woolsey and D. J. BuRns (1967). Absolute cross section for electron impact excitation of $3914 \AA \mathrm{N}_{2}{ }^{+}$, Planet. Space Sci. 15, 1332.

RAPP, D. and P. ENGLANDER-GolDEN (1965). Total cross sections for ionization and attachment in gases by electron impact. I. positive ionization. J. chem. Phys. 43, 1464.

ReEs, M. H. (1963). Auroral ionization and excitation by incident energetic electrons. Planet. Space Sci. $11,1209$.

REes, M. H. (1964). Ionization in the Earth's atmosphere by aurorally associated bremsstrahlung X-rays. Planet. Space Sci. 12, 1093.

Rees, M. H., J. C. G. Walker and A. Dalgarno (1967). Auroral excitation of the forbidden lines of atomic oxygen. Planet. Space Sci. 15, 1097.

StOLARSkI, R. S. and A. E. S. GREEN (1967). Calculations of auroral intensities from electron impact. J. geophys. Res. 72, 3967.

Stolarski, R. S., V. A. Dulock, JR., C. E. Watson and A. E. S. Green (1967). Electron impact cross sections for atmospheric species, 2, molecular nitrogen. J. geophys. Res. 72, 3953.

Watson, C. E., V. A. Dulock, JR., R. S. Stolarski and A. E. S. Green (1967). Electron impact cross sections for atmospheric species, 3, molecular oxygen. J.geophys. Res. 72, 3961. 